\title{
Pyruvate Kinase M2 Mediates Glycolysis in the Lymphatic Endothelial Cells and Promotes the Progression of Lymphatic Malformations
}

\author{
Hao Jiang, ${ }^{* \dagger}$ Yanping Zou, ${ }^{* \dagger}$ Jihong Zhao, ${ }^{* \dagger}$ Xuecong $\mathrm{Li}^{,{ }^{\dagger}}$ Shaodong Yang, ${ }^{\S}$ Xiaoshun Zhou, ${ }^{\ddagger}$ Dongsheng Mou, \\ Wenqun Zhong, ${ }^{* \dagger}$ and $\mathrm{Yu} \mathrm{Cai}^{{ }^{\dagger}}$
}

From The State Key Laboratory Breeding Base of Basic Science of Stomatology (HUbei-MOST) \& Key Laboratory of Oral Biomedicine Ministry of Education, School \& Hospital of Stomatology, ${ }^{*}$ the Department of Oral \& Maxillofacial Surgery, School \& Hospital of Stomatology, ${ }^{\dagger}$ and the Department of Pathology, ${ }^{\S}$ School and Hospital of Stomatology, Wuhan University, Wuhan; and Wuhan Conform Pharm Co., Ltd., Wuhan, China

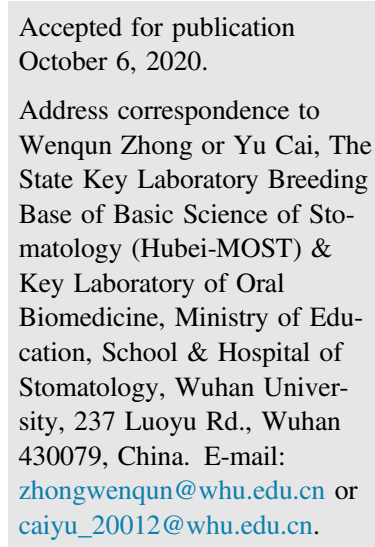

Metabolism plays a pivotal role in the formation of the lymphatic vasculature. Pyruvate kinase M2 (PKM2) is typically a metabolic marker of proliferating cells and maintains the growth of vascular endothelial cells. In this study, the potential status of PKM2 in lymphatic endothelial cells and the pathogenesis of lymphatic malformations (LMs) was investigated. The glycolysis index, including glucose uptake, ATP, and lactate production, stayed at a relatively high level in human dermal lymphatic endothelial cells (HDLECs) compared with human umbilical vein endothelial cells, whereas the inhibition of PKM2 by shikonin or PKM2 knockdown significantly suppressed glycolysis, migration, tubular formation, and invasion of HDLECs. Moreover, compared with lymphatic vessels in healthy skin, lymphatic vessels of LMs expressed PKM2 highly, and this expression correlated with infection of LMs. Meanwhile, the overexpression of PKM2 in HDLECs strengthened the proliferation, migration, tubular formation, and invasion of HDLECs. The findings from further experiments in a rat LM model support that targeting PKM2 by shikonin significantly impedes the progression of LMs, even in an infected LM rat model. Taken together, these results indicate that PKM2 plays a pivotal role in the activation of LECs and promotes the progression of LMs, whereas the inhibition of PKM2 can effectively suppress the pathogenesis of LM lesions in the rat model. (Am J Pathol 2021, 191: 204-215; https://doi.org/10.1016/j.ajpath.2020.10.003)
Glycolysis, an energy-metabolic process, is the conversion of glucose into pyruvate by a series of rate-limiting glycolytic enzymes, which normally occurs in anerobic or low-oxygen conditions. However, as noted by Warburg in the 1920s, cancer cells exert a high rate of glycolysis even in the presence of abundant oxygen, known as aerobic glycolysis or the Warburg effect. ${ }^{1}$ Further investigations found that the Warburg effect not only occurred in cancer cells but also in rapidly proliferating cells. Despite lesser ATP generation compared with oxidative phosphorylation, the up-regulated glycolysis meets the metabolic requirements of cell proliferation by affording adequate energy and sufficient glycolytic intermediates for macromolecular synthesis (eg, amino acids, phospholipids, and nucleic acids). ${ }^{2,3}$ Moreover, the findings from a previous study ${ }^{4}$ showed that the glycolysis level in endothelial cells (ECs) was much higher than that in other noncancer type of cells and largely comparable to that in tumor cells. EC metabolism has recently emerged as an important regulator of angiogenesis and lymphangiogenesis. In both developmental processes, glycolysis is the main source of energy production and drives EC migration and proliferation. ${ }^{5}$ The importance of rate-limiting glycolytic enzymes, such as phosphofructokinase-2/fructose-2,6-bisphosphatase

\footnotetext{
Supported by National Natural Science Foundation of China (NSFC) grants 81741082 (Y.C.), 81800994 (W.Z.), and 81671008 (J.Z.); National Health Commission of the People's Republic of China grant 2019ZX09302011 (D.M. and Y.C.); and Wuhan Science and Technology Bureau grant 2020020601012212 (Y.C.).

H.J. and Y.Z. contributed equally to this work.

Disclosures: X.Z. and D.M. are employees of Wuhan Conform Pharma Co., Ltd.
} 
(PFKFB)-3 or hexokinase (HK)-2, on angiogenesis has been reported. $^{6}$

Pyruvate kinase $(\mathrm{PK})$ plays a role in as the final step in glycolysis, transferring a phosphate group from phosphoenol pyruvate to ADP, thereby generating pyruvate and ATP. ${ }^{2}$ This makes PK ideal for regulating glycolytic flux. Of the four PK isoforms in mammals (L, R, M1, and M2), PK type M2 (PKM2) is a characteristic isozyme in fastproliferating cells, such as cancer cells, embryonic cells, and stem cells. ${ }^{7}$ In many cancer cells, abnormal elevation of PKM2 activity and tetramer/dimer ratio could adversely exacerbate the metabolism, proliferation, and tumorigenic capacity of cancer cells. ${ }^{7}$ Analogously, in vascular ECs, PKM2 mediated sprouting angiogenesis by overactive glycolysis could provides material and energy that promotes the EC-junction dynamics, collective migration, and proliferation. ${ }^{6}$ However, its effects on lymphatic ECs (LECs) and lymphangiogenesis have not been reported.

Lymphatic malformations (LMs) are congenital, arising from disordered lymphatic vessels in the embryo. ${ }^{8}$ The findings from previous studies support the involvement of abnormal proliferation of LECs and lymphangiogenesis in the pathogenesis of LMs. ${ }^{8,9}$ Despite abundant evidence regarding the association between PKM2-mediated glycolysis and rapidly proliferating disease, few studies have determined its significance in LMs. Therefore, in the present study, the role of PKM2 in LECs and lymphangiogenesis, as well as its potential effect in LMs, were investigated.

\section{Materials and Methods}

\section{Cell Culture}

Human dermal lymphatic endothelial cells (HDLECs) (catalog number 2010; ScienCell, Carlsbad, CA) and human umbilical vein endothelial cells (HUVECs) were cultured according to a previous study. ${ }^{10,11}$

\section{Glucose Uptake, Lactate, and ATP Production}

After treatment for 24 hours, lactate in the culture media, intracellular glucose, and ATP were measured using the Lactic Acid Assay Kit (catalog number A019-2-1; Nanjing Jiancheng Bioengineering Institute, Nanjing, China), Glucose Uptake Cell-Based Assay Kit (catalog number 600470; Cayman Chemical, Ann Arbor, MI), and the ATP Assay Kit (catalog number A095-1-1; Nanjing Jiancheng Bioengineering Institute), respectively, according to the manufacturers' instructions.

\section{Transfection and Treatments}

The infection of HDLECs with lentiviral vector-loaded shRNAs against human PKM2 was performed for the knockdown of PKM2. Briefly, shRNAs targeting sequences for PKM2 was 5'-CTACCACTTGCAATTATTTGA-3'. The
shRNAs against PKM2, scrambled shRNA-control (Addgene, Watertown, MA), were packaged into lentiviral particles by using $293 \mathrm{~T}$ cells. Lentiviral supernatants containing lentiviral particles were then harvested with 72-hour culture after transfection. HDLECs were then infected with lentivirus and selected using $2 \mu \mathrm{g} / \mathrm{mL}$ puromycin. After 24-hour transfection, efficiency was validated with PKM2 expression using Western blot analysis.

Lentiviral vector-loaded Flag-PKM2 was used for inducing overexpression of PKM2 in HDLEC. Briefly, PCR-amplified human PKM2 was cloned into pCDH-Flag. Then, the pCDH-Flag-PKM2 or pCDH-Flag were packaged into lentiviral particles with 293T cells. After incubation for 72 hours, the cell culture supernatants were harvested. HDLECs in the logarithmic stage were plated with a density of $1 \times 10^{5}$ cells per $6-\mathrm{cm}$ dish and incubated for 48 hours at $37^{\circ} \mathrm{C}, 5 \% \mathrm{CO}_{2}$. While cells were $80 \%$ to $90 \%$ polymerized, and lentivirus supernatant was added and screened using 2 $\mu \mathrm{g} / \mathrm{mL}$ puromycin. After 24-hour transfection, efficiency was validated with PKM2 expression using Western blot analysis.

\section{Western Blot Analysis}

As previously reported, ${ }^{12}$ the whole-cell lysate was collected and the concentration of protein was determined by a bicinchoninic acid protein assay kit (catalog number P0012S; Beyotime, Shanghai, China). A total of $25 \mu \mathrm{g}$ of protein from each group was separated on 10\% SDSpolyacrylamide gels and was wetly transferred on polyvinylidene difluoride membrane by electrophoresis. Subsequently, the membrane was blocked by 5\% skim milk on an oscillating table at room temperature for 1 hour and then incubated with targeted protein antibodies PKM2 (1:1000; catalog number ab137852; Abcam, Cambridge, UK) and $\beta$-actin (1:2000; catalog number ab8227; Abcam) overnight at $4^{\circ} \mathrm{C}$. The blots were further incubated with horseradish peroxidase-conjugated secondary antibodies at room temperature for 1 hour, then detected using a chemiluminescence kit (Thermo Fisher Scientific, Waltham, MA) and imaged for records.

\section{Cell Proliferation, Migration, Invasion, and Tubular Formation Assay}

The MTT assay, wound-healing assay, transwell invasion assay, and tubular formation assay were respectively performed to evaluate cell proliferation, migration, invasion, and tubular formation in different pretreated conditions according to a previous study. ${ }^{10}$ For MTT assay, HDLECs were seeded in 96-well plates $\left(2 \times 10^{4}\right.$ cells/well $)$ in quintuplicate. After cell attachment, culture medium was replaced with medium containing shikonin (catalog number 517-89-5; Selleck, Shanghai, China; $0.0,0.2,0.5,1.0,2.0,5.0$, and $10.0 \mu \mathrm{mol} / \mathrm{L}$ ) for appropriate time ( 24 or 48 hours). Then, $20 \mu \mathrm{L}$ of MTT $(5 \mathrm{mg} / \mathrm{mL})$ solution was added directly to each well and 
treated for 4 hours at $37^{\circ} \mathrm{C}$. After the medium was removed, the synthetic insoluble MTT formazan was dissolved in 150 $\mu \mathrm{L}$ of dimethyl sulfoxide and quantified using spectrophotometry at a wavelength of $570 \mathrm{~nm}$.

Before wound-healing assay, invasion assay, and tubular formation assay, all HDLECs used in the studies were pretreated with shikonin $(0,0.2$, or $0.5 \mu \mathrm{mol} / \mathrm{L})$ for 6 hours. For wound-healing assay, $5 \times 10^{5}$ cells/well were seeded into 6-well plates in quintuplicate and grown until $90 \%$ confluence. The monolayer of cells was scratched with a $10-\mu \mathrm{L}$ sterilized pipette tip, washed with phosphate-buffered saline for the removal of debris, incubated with fresh serumfree endothelial cell medium (ECM) (catalog number 1001; ScienCell) medium and cultivated under standard conditions. The images of HDLEC migration were recorded with an inverted microscope. The capacity of migration through Matrigel was evaluated using transwell invasion assay. The upper chambers were precoated with $100 \mu \mathrm{L}$ of Matrigel (catalog number 356234; Becton, Dickinson and Co., Franklin Lakes, NJ) for 1 hour at $37^{\circ} \mathrm{C}$, followed by the spread of a total of $2 \times 10^{4}$ cells into the upper chamber with serum-free ECM medium in quintuplicate. The lower chamber was filled with $600 \mu \mathrm{L}$ of extracellular matrix medium containing 5\% fetal bovine serum (FBS) (catalog number 0025; ScienCell), which was the provenience of chemoattractant. After 24 hours, cells that had traversed the membrane were fixed with $4 \%$ phosphate-buffered paraformaldehyde for 20 minutes and stained with crystal violet. After drying at room temperature, five visual fields were randomly chosen for further statistical analysis. As to tubular formation assay, $2 \times 10^{5}$ pretreated HDLECs were seeded onto a Matrigel-coated 48-well plate. After 4 hours, the samples were fixed with $4 \%$ paraformaldehyde and the number of capillary-like structures was calculated.

\section{Patients and Tissue Samples}

Human LM tissues $(n=25)$ and healthy skin tissues $(n=8)$ were collected from the Hospital of Stomatology, Wuhan University (Wuhan, China). The clinical characteristics of the patients are presented in Supplemental Tables S1 and S2. Informed consent was signed by all patients after detailed explanation. The study protocol and sample collection were approved by the review board of the Ethics Committee of the Hospital of Stomatology, Wuhan University.

\section{IHC Analysis, Double-Labeling Immunofluorescence, and Evaluation}

Immunohistochemistry staining was performed as previously reported. ${ }^{10}$ Briefly, formalin-fixed paraffinembedded specimens from human LM tissues and healthy skin tissues were deparaffinized with xylene and rehydrated in ethanol gradient. After antigen retrieval and peroxidase blocking, the slices were incubated with specific primary antibodies PKM2 (1:200; catalog number ab137852; Abcam), Ki-67 (1:400; catalog number ab245113; Abcam), prospero homeobox protein 1 (PROX-1) (1:200; catalog number 14963; Cell Signaling Technology, Boston, MA) and podoplanin (ready to use; catalog number ZM-0465; ZSGB-Bio, Beijing, China) at $4^{\circ} \mathrm{C}$ overnight. After that, horseradish peroxidase-conjugated secondary antibody was applied to bind to primary antibodies, and the binding was detected and visualized with a DAB substrate kit (catalog number 0017; MXB Biotechnologies, Fuzhou, China).

Protein expression was quantified with visual criteria based on the staining intensity (grade scale: 0 , negative; 1, mild staining; 2, moderate staining; or 3, strong staining) and the proportion of positive-stained LECs/ podoplanin ${ }^{+}$and PROX $-1^{+}$LECs (grade scale: $0,<10 \%$; $1,10 \%$ to $<25 \% ; 2,25 \%$ to $<50 \% ; 3,50 \%$ to $<75 \%$; or 4 , $\geq 75 \%$ ). The evaluation of immunohistochemistry staining was performed by two blinded investigators (H.J. and Y.Z.).

Double-labeling immunofluorescence was performed to exhibit the co-expression of PKM2 and PROX-1, Ki-67 and PROX-1, PKM2 and Ki-67 in healthy skin samples and LMs. After dewaxing and hydrating of specimens, the antigen was retrieved using high temperature. Then all samples were incubated with $10 \%$ nonimmune goat serum for 1 hour at $37^{\circ} \mathrm{C}$. Subsequently, the sections were placed at $4{ }^{\circ} \mathrm{C}$ overnight with primary antibodies: PKM2 (1:100), PROX-1 (1:100), and Ki-67 (1:100; catalog number 9449; Cell Signaling Technology). After the incubation of secondary antibodies DyLight 488 or DyLight 594, DAPI was applied to mark the nuclei. Finally, the images were captured using a fluorescence microscope (Leica Microsystems, Buffalo Grove, IL).

\section{Establishment of the LM Model in Rats}

Female Wistar rats (weight, $250 \pm 30 \mathrm{~g}$ ) were purchased from the Experimental Animal Center (Hubei Province, China). All rats received appropriate care according to the requirements of the Ethics Committee for Animal Research, Wuhan University. The Freund's incomplete adjuvant (FIA, catalog number F5506; Sigma-Aldrich, Shanghai, China) induced LM rat model was established as presented previously $^{9,10,13}$; FIA and lipopolysaccharide (LPS; catalog number L4391; Sigma-Aldrich, St. Louis, MO) was used for establishing the infected LM rat model. ${ }^{9}$ In brief, LM rat models were established with two s.c. injections (2 weeks apart) of FIA in subcutaneous of the neck. Four weeks later, LM rat models were established successfully, with most lesions size of 250 to $300 \mathrm{~mm}^{3}$ and then randomly classified into four different treatment groups: i) $200 \mu \mathrm{L}$ PBS, ii), 200 $\mu \mathrm{L}$ PBS containing $200 \mu \mathrm{g} / \mathrm{mL}$ shikonin, iii) $200 \mu \mathrm{L}$ PBS containing $100 \mu \mathrm{g} / \mathrm{mL}$ LPS, iv) $200 \mu \mathrm{L}$ PBS containing 100 $\mu \mathrm{g} / \mathrm{mL}$ LPS and $200 \mu \mathrm{g} / \mathrm{mL}$ shikonin. In the subsequent 8 weeks, LM lesions were treated with different drug administrations every other day and the volumes were measured 
A

B
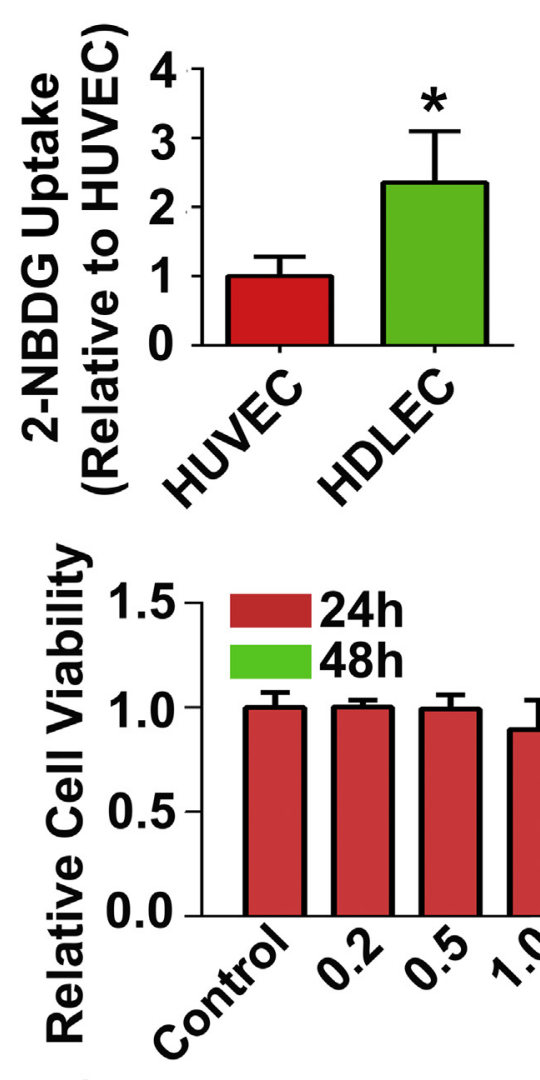
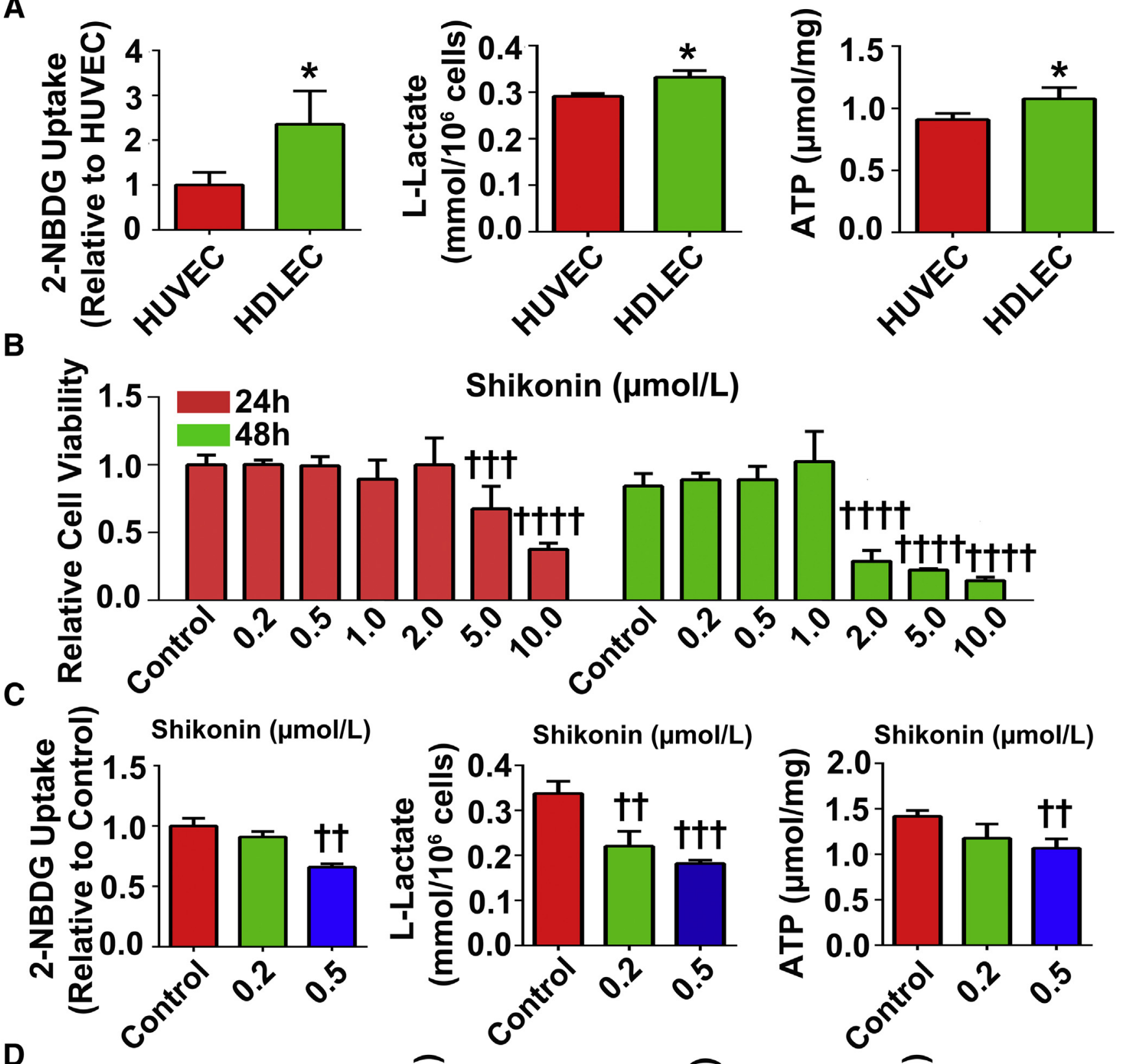

Shikonin ( $\mu \mathrm{mol} / \mathrm{L})$
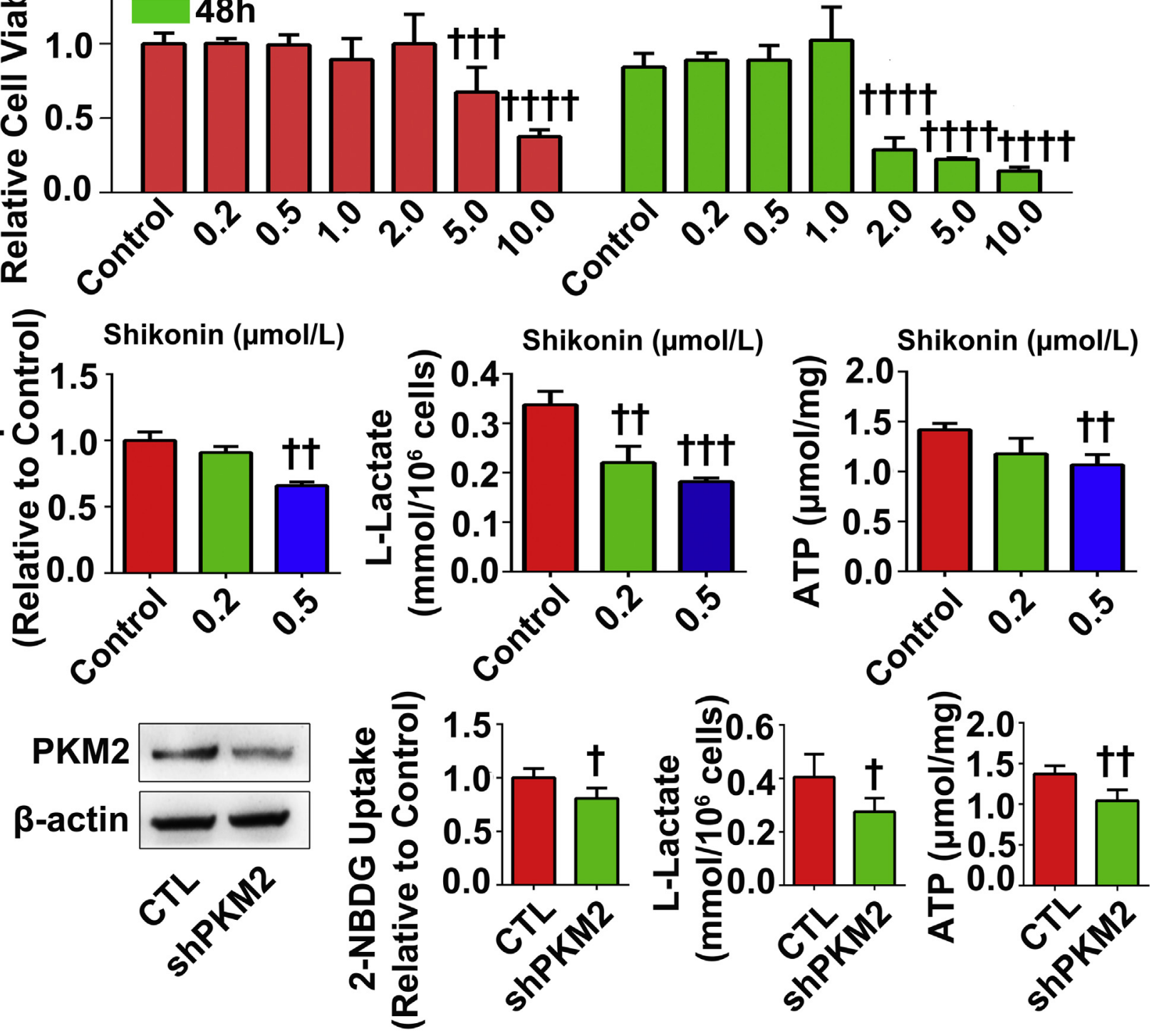

Figure 1 PKM2 is necessary for the glycolysis of human dermal lymphatic endothelial cells (HDLECs). A: Glucose uptake, lactate, and ATP production are significantly elevated in HDLECs compared with that in HUVECs. B: MTT assay shows that the proliferative activity of HDLECs is not inhibited with treatment with $\leq 1.0 \mu \mathrm{mol} / \mathrm{L}$ shikonin within 48 hours. C: The glycolysis indexes of HDLECS, including glucose uptake, ATP, and lactate production capacity, are markedly decreased with $0.5 \mu \mathrm{mol} / \mathrm{L}$ shikonin treatment for 24 hours. D: After knockdown of the expression of PKM2, the glycolysis indexes of HDLECs are down-regulated. Data are expressed as means \pm SD. ${ }^{*} P<0.05$ versus HUVEC; ${ }^{\dagger} P<0.05,{ }^{\dagger \dagger} P<0.01,{ }^{\dagger \dagger \dagger} P<0.001$, and ${ }^{\dagger \dagger \dagger \dagger} P<0.0001$ versus control. 2-NBDG, 2-deoxy-2-[(7-nitro-2,1,3-benzoxadiazol-4-yl) amino]-D-glucose; CTL, control. 

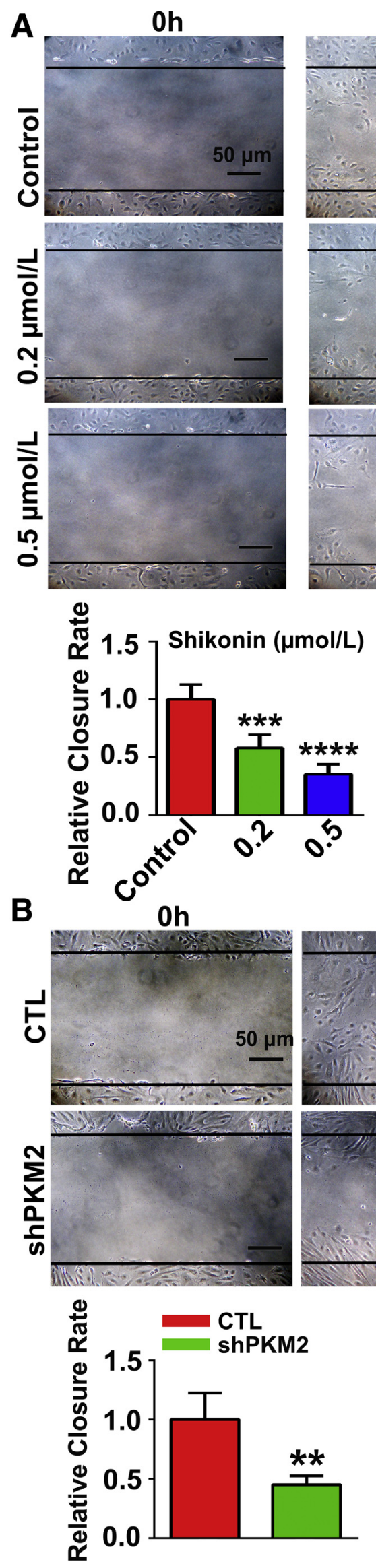

$24 h$
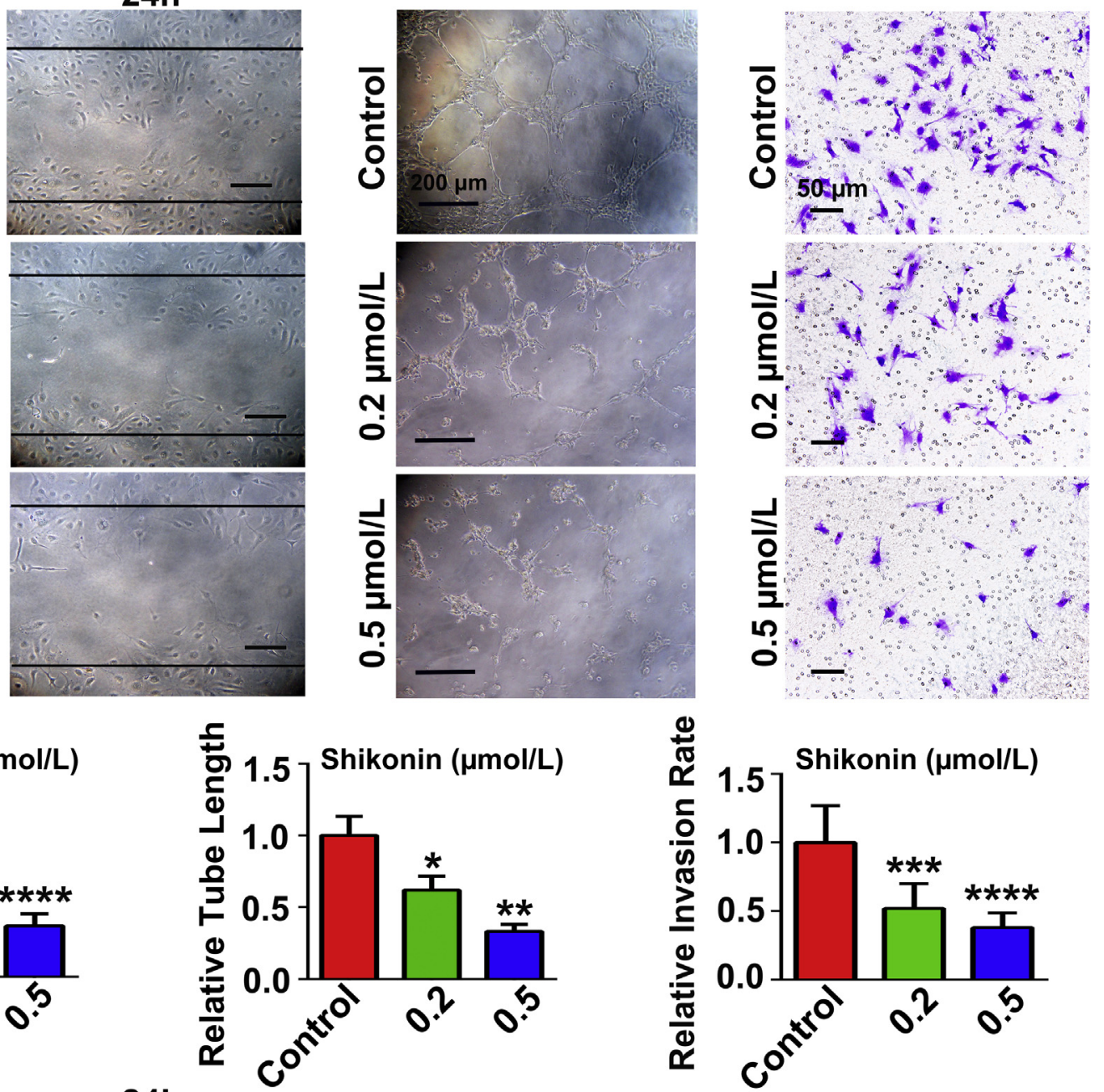

24h
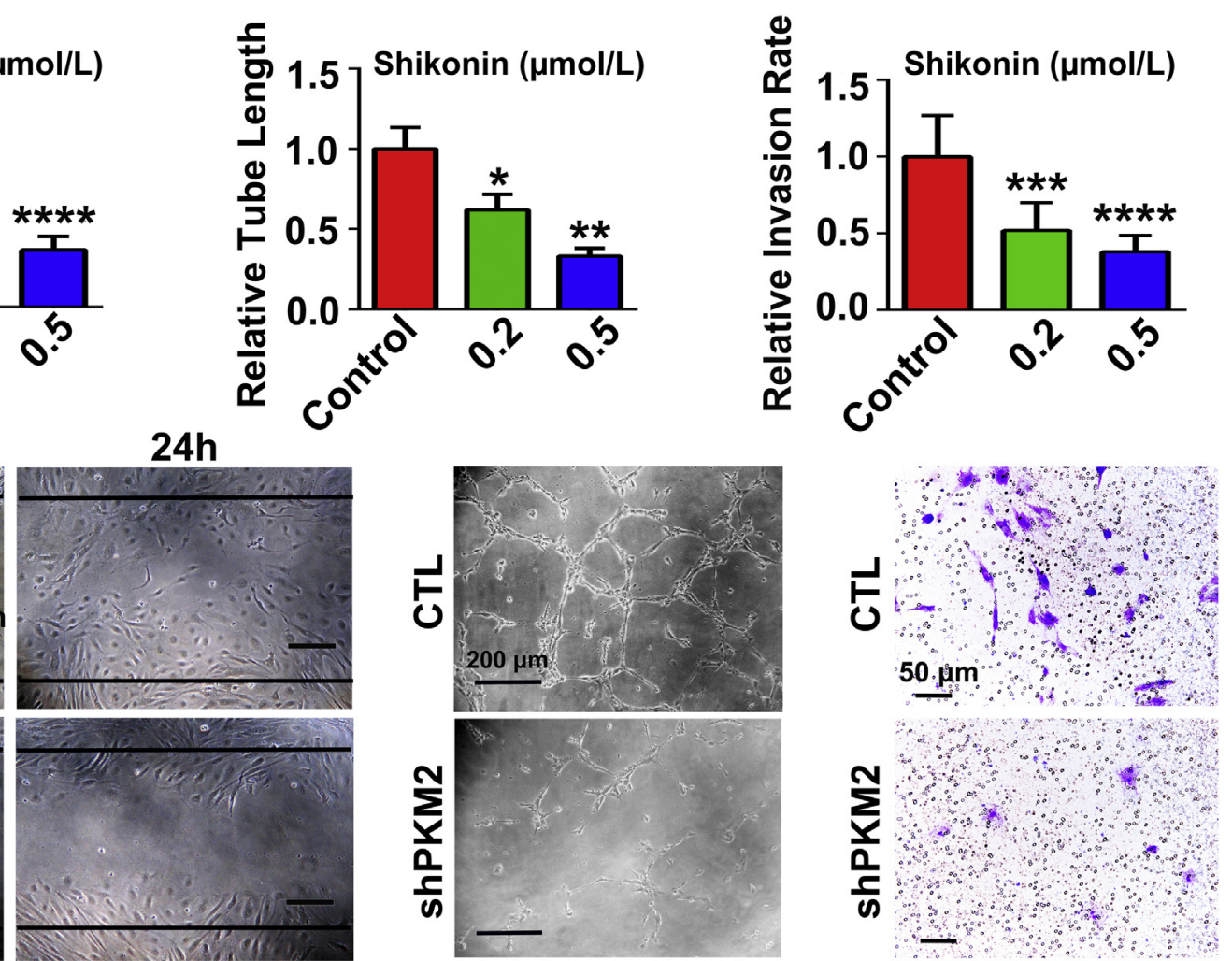

$\frac{\sum^{\frac{N}{n}}}{\frac{\mathbf{x}}{n}}$

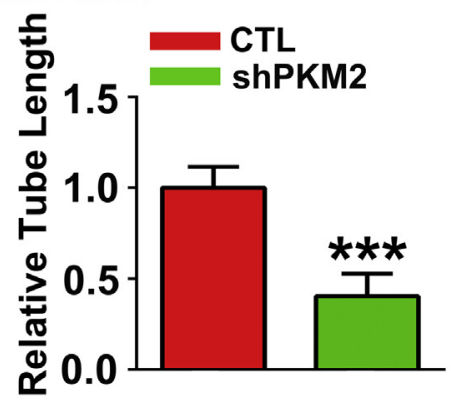


each week. Then all of the rats were euthanized, and the LM lesions were collected, measured, and weighed for analysis. The lesions were used for further experiments.

\section{Statistical Analysis}

Statistical analyses were performed with Prism software version 6.0 (GraphPad Software, Inc.) for statistical analysis. The associations between PKM2 expression and clinical factors were analyzed using the $\chi^{2}$ test. The Spearman rank correlation coefficient test and linear tendency test were applied for analysis of the correlation between the expression of PKM2 and Ki-67. The $t$-test was used for evaluating the differences between the experimental and control groups. All data are expressed as the means \pm SD from three independent experiments. $P<0.05$ was considered statistically significant.

\section{Results}

\section{PKM2 Is Necessary for Glycolysis of HDLECs}

Since human umbilical vein ECs have higher glycolysis rates compared with many noncancer type of cells, ${ }^{6}$ HUVECs were used as a positive control for the detection of glycolysis in HDLECs. Glucose uptake, lactate, and ATP production were significantly elevated in HDLECs compared with that in HUVECs (Figure 1A), which indicates extremely high glycolysis in HDLECs.

Highly activated glycolysis was required for supporting cell proliferation by supplying glycolytic intermediates for the synthesis of macromolecules in addition to generating ATP. ${ }^{2}$ PKM2 plays a vital role in tumor metabolism and can be potentially regarded as a chemotherapeutic drug target for cancer therapy. ${ }^{7}$ Recently, shikonin, a naphthoquinone extracted from the root of purple perilla, was identified as a major inhibitor of PKM2 by its inhibition of phosphorylation and, eventually, reduction of glycolysis in cancer cells. ${ }^{14-16}$ Therefore, in the present study, shikonin was used for exploring the role of PKM2 in HDLEC glycolysis. Glycolysis indexes of HDLECs, including glucose uptake, ATP, and lactate production capacity were markedly decreased with shikonin treatment (Figure 1C), even at a concentration at which it had no significant effect on cell viability (Figure 1B). For further investigating the role of PKM2, shRNA against PKM2 was constructed for inhibiting the expression of PKM2 in HDLECs. Knockdown of PKM2 resulted in the downregulation of the glycolysis indexes of HDLECs (Figure 1D). These results are consistent with PKM2-mediated inhibition of aerobic glycolysis in tumor cells. ${ }^{16}$
PKM2 Is Necessary for the Migration, Tubular Formation, and Invasion of HDLECs

Shikonin-treated HDLECs were used to investigate the role of PKM2 in the migration, tubular formation, and invasion of HDLECs. The migration, tubular formation, and invasion potential of HDLECs was inhibited with shikonin treatment in a dose-dependent manner (Figure 2A). Meanwhile, knockdown of PKM2 expression in HDLECs also significantly reduced cell migration, tubular formation, and invasion (Figure 2B). These results indicate that the PKM2-mediated glycolysis plays a critical role in LEC activation and lymphangiogenesis.

Lentiviral vector-loaded Flag-PKM2 was used for overexpressing PKM2 and investigating the function of HDLECs. Overexpression of PKM2 significantly promoted the activity of HDLECs, including proliferation, migration, tubular formation, and invasion (Figure 3).

PKM2 Is Highly Expressed in the Lymphatic Vessels of LMs and Its Expression Is Correlated with Infection of LMs

Previous findings have validated the involvement of abnormal lymphangiogenesis in the pathogenesis of LMs. ${ }^{8,9}$ Due to the significance of PKM2 in lymphangiogenesis, the expression levels of PKM2 were evaluated in a set of $25 \mathrm{LM}$ samples and 8 skin samples from healthy donors. The basic information on the LM patients and healthy donors is shown in Supplemental Tables S1 and S2. The $\chi^{2}$ test showed that there was no significant correlation between PKM2 expression and clinical factors, such as age, sex, and lesion location. Based on previous studies, ${ }^{9,10}$ PROX-1 and podoplanin were used for marking lymphatic vessels. Beginning at embryonic day 9.5 in mice, PROX-1 expressed and controlled the mechanisms specifying lymphatic EC fate. ${ }^{17}$ On day embryonic $11.5 \mathrm{E}$, podoplanin first appeared in the developing circulatory system on PROX-1 ${ }^{+}$lymphatic cells. ${ }^{18,19}$ In the present investigation, both podoplanin ${ }^{+}$and PROX $-1^{+}$vessels were regarded as lymphatic vessels. The expression of PKM2 in the lymphatic vessels was higher in LMs compared with that in healthy skin (Figure 4 and Supplemental Figure S1A). Moreover, in LM samples, staining of PKM2 in the lymphatic vessels was significantly stronger with inflammatory cell infiltration compared to LMs without inflection (Figure 4).

Spearman rank correlation showed a positive correlation between the protein level of PKM2 and Ki-67 in LMs (Figure 5A). PKM2 and Ki-67 co-expressed in the LECs of

\footnotetext{
Figure 2 Inhibition of PKM2 attenuates migration, tubular formation, and invasion of human dermal lymphatic endothelial cells (HDLECS). A: Representative images of HDLEC migration, tubular formation, and invasion with pretreatment with 0.2 or $0.5 \mu \mathrm{mol} / \mathrm{L}$ shikonin for 6 hours. B: Representative images of HDLEC migration, tubular formation, and invasion after knockdown of PKM2 expression. Lines in migration assays indicate the starting point of migration after scratched. Data are expressed as means \pm SD. ${ }^{*} P<0.05,{ }^{*} P P<0.01,{ }^{* * *} P<0.001$, and ${ }^{* * * * P}<0.0001$ versus control. Scale bars: $50 \mu \mathrm{m}$ (left and right columns); $200 \mu \mathrm{m}$ (middle columns). CTL, control.
} 
A

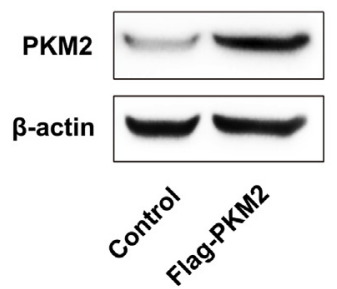

B

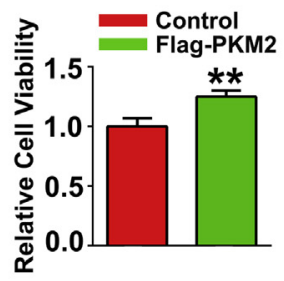

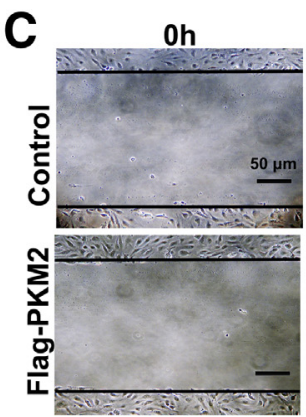
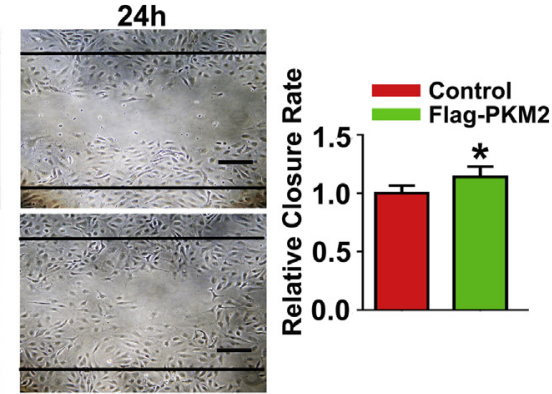
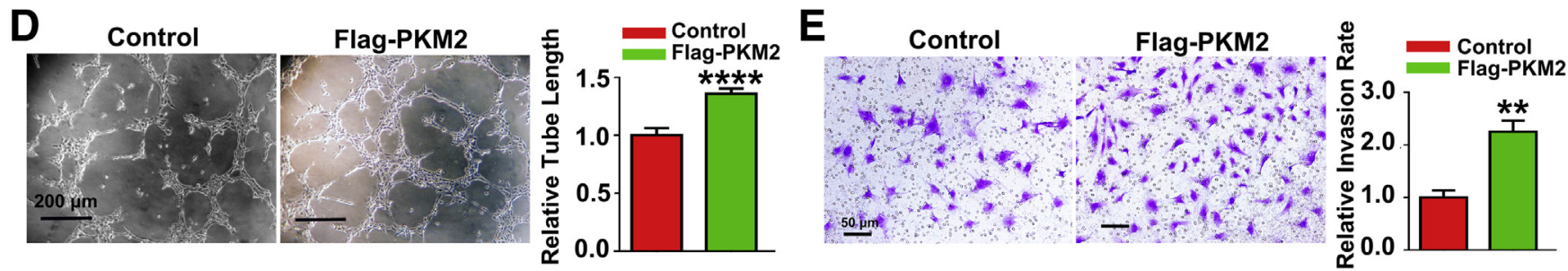

Figure 3 Up-regulation of PKM2 promotes proliferation, migration, tubular formation, and invasion of human dermal lymphatic endothelial cells (HDLECs). A: Results from Western blot analysis show the expression of PKM2 in HDLECs by transfection of the lentiviral vector-loaded Flag-PKM2. B-E: The MTT assay (B), wound healing assay (C), Matrigel tubular formation assay (D), and Transwell assay (E) show proliferation, migration, tubular formation, and invasion of HDLECs with the up-regulation of PKM2. Lines in $\mathbf{C}$ indicate the starting point of migration after scratch. Data are expressed as means \pm SD. ${ }^{*} P<0.05$, ${ }^{* *} P<0.01$, and ${ }^{* * * * P}<0.0001$ versus control. Scale bars: $50 \mu \mathrm{m}$ (C and $\left.\mathbf{E}\right) ; 200 \mu \mathrm{m}$ (D).

LMs but not in healthy skin, as analyzed using double-stain immunofluorescence (Figure 5B). These results indicate that the overexpression of PKM2 may accelerate the progression of LMs.

\section{Inhibition of PKM2 Suppresses the Formation of LM Lesions in a Rat Model}

To further verify the role of PKM2 in the progression of LMs, a rat model of LM was constructed according to a previous study. ${ }^{9}$ After two s.c. injections of FIA (2 weeks apart), the rats received different treatments every other day for 8 weeks, and were euthanized for the collection of the lesions. Figure 6A shows the typical lesions in the neck of the rat. Lesion volumes and weights support the therapeutic effect of shikonin in the lesions both with and without infection (Figure 6B). The body weights (Figure 6C) and important organic tissues stained with hematoxylin and eosin (Figure 6D) after treatment were comparable to those of the control group, indicating the safety of the drug administration. Furthermore, immunohistochemistry staining of Ki-67, vascular endothelial growth factor C (VEGFC), and PKM2 was used for detecting proliferation, lymphangiogenesis, and PKM2 expression in LECs in the LM rat samples, respectively (Figure 7). Though the protein levels of Ki-67, VEGF$\mathrm{C}$ and PKM2 were up-regulated with LPS, they were severely down-regulated with shikonin, which offset the effect of LPS. Therefore, targeting PKM2 by its selective suppressant shikonin significantly impeded the progression of LMs, even in the infected condition, in the rat model of LM.

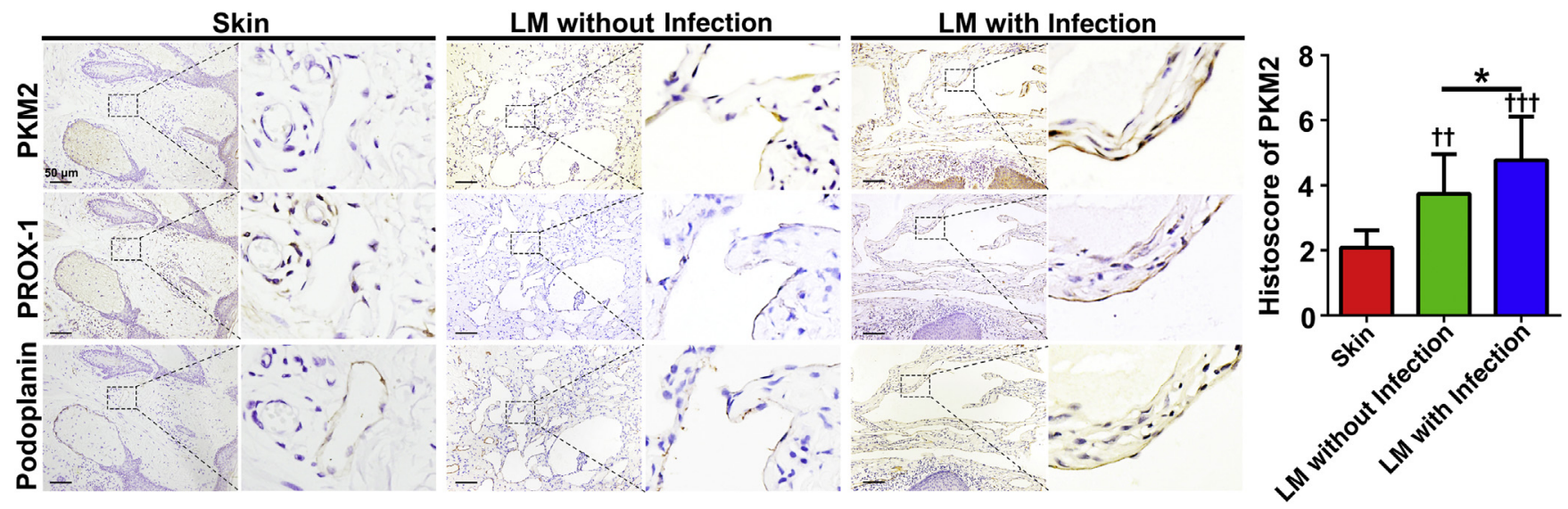

Figure 4 Serial section staining for PKM2, PROX-1, and podoplanin in lymphatic vessels of normal skins and LM samples. Data are expressed as means \pm SD. ${ }^{*} P<0.05 ;{ }^{\dagger \dagger} P<0.01$, and ${ }^{\dagger \dagger} P<0.001$ versus skin. Scale bars $=50 \mu \mathrm{m}$. 

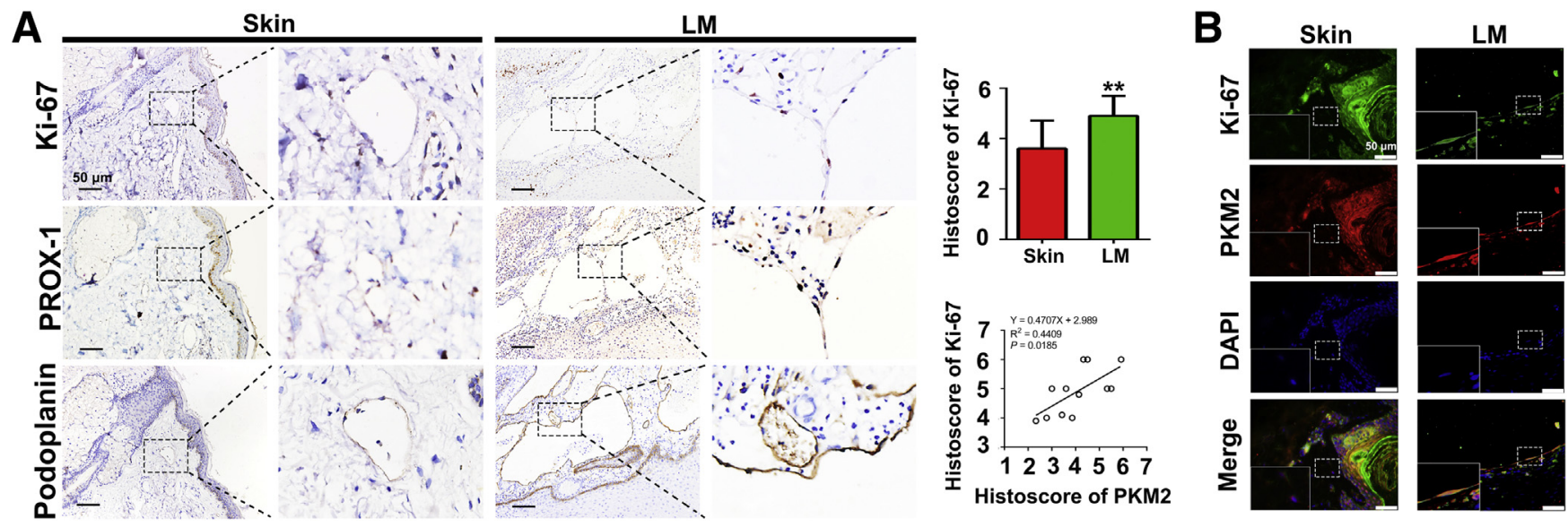

Figure 5 A: Serial section staining for Ki-67, prospero homeobox protein (PROX)-1 and podoplanin in lymphatic vessels of healthy skin tissue and LM samples. Spearman rank correlation shows that the protein level of PKM2 is positively correlated with Ki-67 in LMs. B: Double-labeling immunofluorescence analyses for PKM2 and Ki-67 shows that these two proteins are co-expressed in the lymphatic vessels of LMs. Boxed areas are shown at higher magnification in the insets. Data are expressed as means \pm SD. ${ }^{* *} P<0.01$ versus skin. Scale bars $=50 \mu \mathrm{m}$.

\section{Discussion}

Warburg's finding that tumor cells express high level of glycolysis in the presence of abundant oxygen offers novel insight into the role of glycolysis in proliferating cells. The up-regulated glycolysis in fast-proliferating cells can provide adequate energy for cell growth and glycolytic intermediates for biosynthetic building blocks, thereby facilitating the assembly of macromolecules and organelles essential for a new cell. ${ }^{2}$ In both lymphangiogenesis and angiogenesis, the assembly of these vascular networks involving sprouting, proliferation, and migration of ECs, require high energy. ${ }^{3,20}$ During these processes, the proliferating ECs undergo vast transendothelial transport to perivascular cells, less oxidative stress, and continue sprouting even in hypoxia. ${ }^{12}$ Therefore, ECs rely heavily on glycolysis for completing these processes, including proliferation, migration, and tubular formation.

In highly proliferative ECs, energy consumption meets the requirements of not only maintaining homeostasis, but also growth and division. Assembling a new daughter cell demands the replication of all cellular components, including proteins, DNA, and RNA. Glycolysis converts glucose into the biosynthetic building blocks and ATP, which ensures energy and supply of raw materials needed for supporting macromolecular synthesis and cell division. The extracellular lactate, which increased with elevated glycolytic flux, was considered crucial for EC motility via the activation of the VEGF-VEGF receptor 2 axis. ${ }^{21}$ In return, during vessel sprouting, the activation of VEGFR2 in stalk cell up-regulated metabolic enzymes and increased glycolysis. ${ }^{22}$ Note that glycolytic enzymes and ATP are localized and concentrated in the lamellipodia and filopodia of ECs, suggesting that glycolysis mediates the fast motion of ECs. ${ }^{20}$ Several glycolytic enzymes, including PFKFB-3 and HK-2, have been found in the regulatory process of angiogenesis or lymphangiogenesis. . $^{4,23}$

PKM2, the final irreversible glycolytic rate-limiting enzyme, is a gatekeeper between cell growth and survival. $^{25}$ Although the importance of glycolysis in lymphangiogenesis has been well-established, ${ }^{23}$ the role of PKM2 in LEC biology has not been thoroughly examined. In the present study, shikonin, a PKM2 inhibitor, was associated with reduced glucose uptake and lactate and ATP production. These results suggest that PKM2 plays a crucial role in the glycolysis of LECs. Meanwhile, with the inhibition of PKM2 with shikonin or knockdown of PKM2, glycolysis was significantly reduced along with proliferation, migration, and tubular formation of LECs, and were enhanced with the overexpression of PKM2 in LECs. These results indicate the significance of PKM2 in lymphangiogenesis. Therefore, we were interested to explore whether PKM2 involves the pathogenesis of LMs, which is characteristic of abnormal lymphangiogenesis and LEC proliferation.

LMs are congenital, arising from disordered lymphatic vessels in the embryo. ${ }^{8}$ Histologically, the lesions are composed of abnormally formed, varying-size lymphatic channels lined in a single layer of flattened endothelium and filled with eosinophilic, protein-rich fluid. The walls of channels consist of abnormally formed skeletal muscular and smooth elements. ${ }^{9}$ With a prevalence of $1.2 \%$ to $2.8 \%$ in neonates and $75 \%$ of lesions occurs in the head and neck region, LMs often are evident at birth or in childhood, tend to expand during adolescence, and enlarge rapidly with infection or trauma. The enlarged mass can damage vital organs, destroy bone, cause disfigurement, or even be life threatening. ${ }^{8}$ Though sclerotherapy and surgical resection 
A

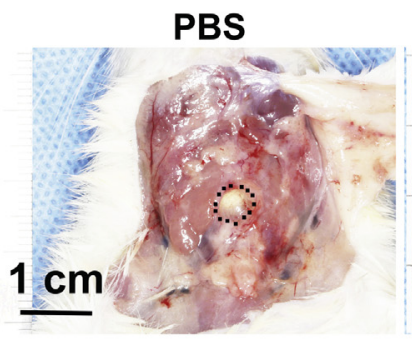

B

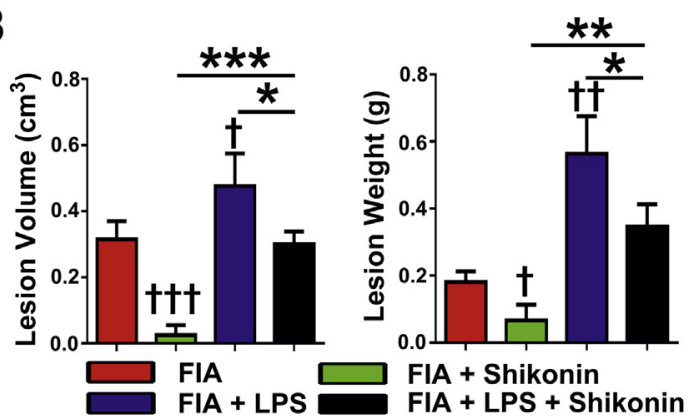

D
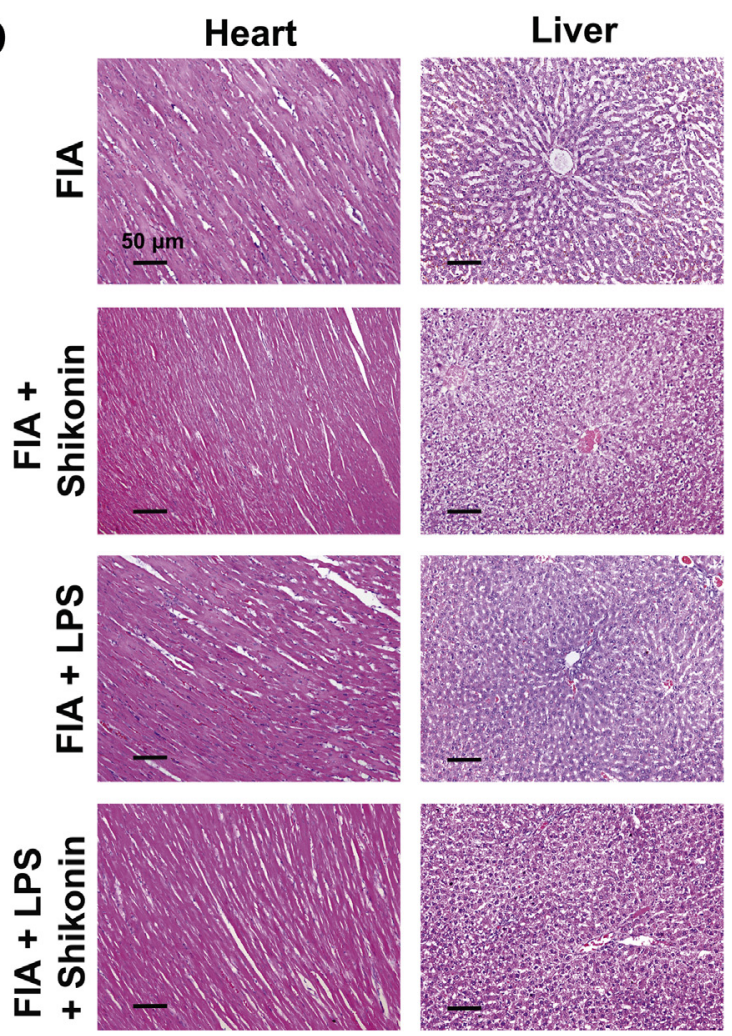
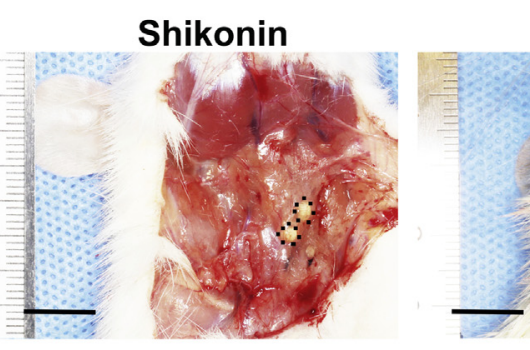
LPS
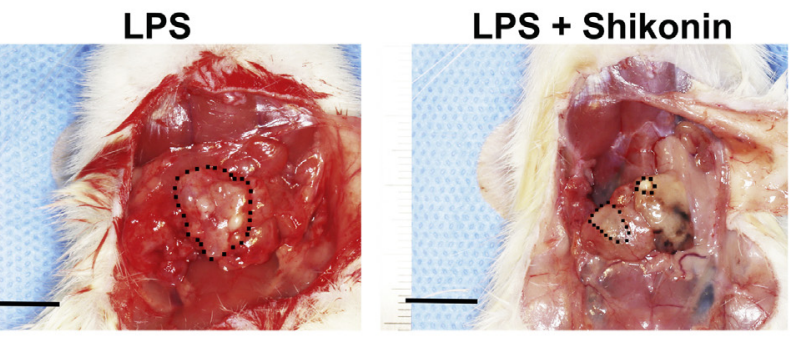

C
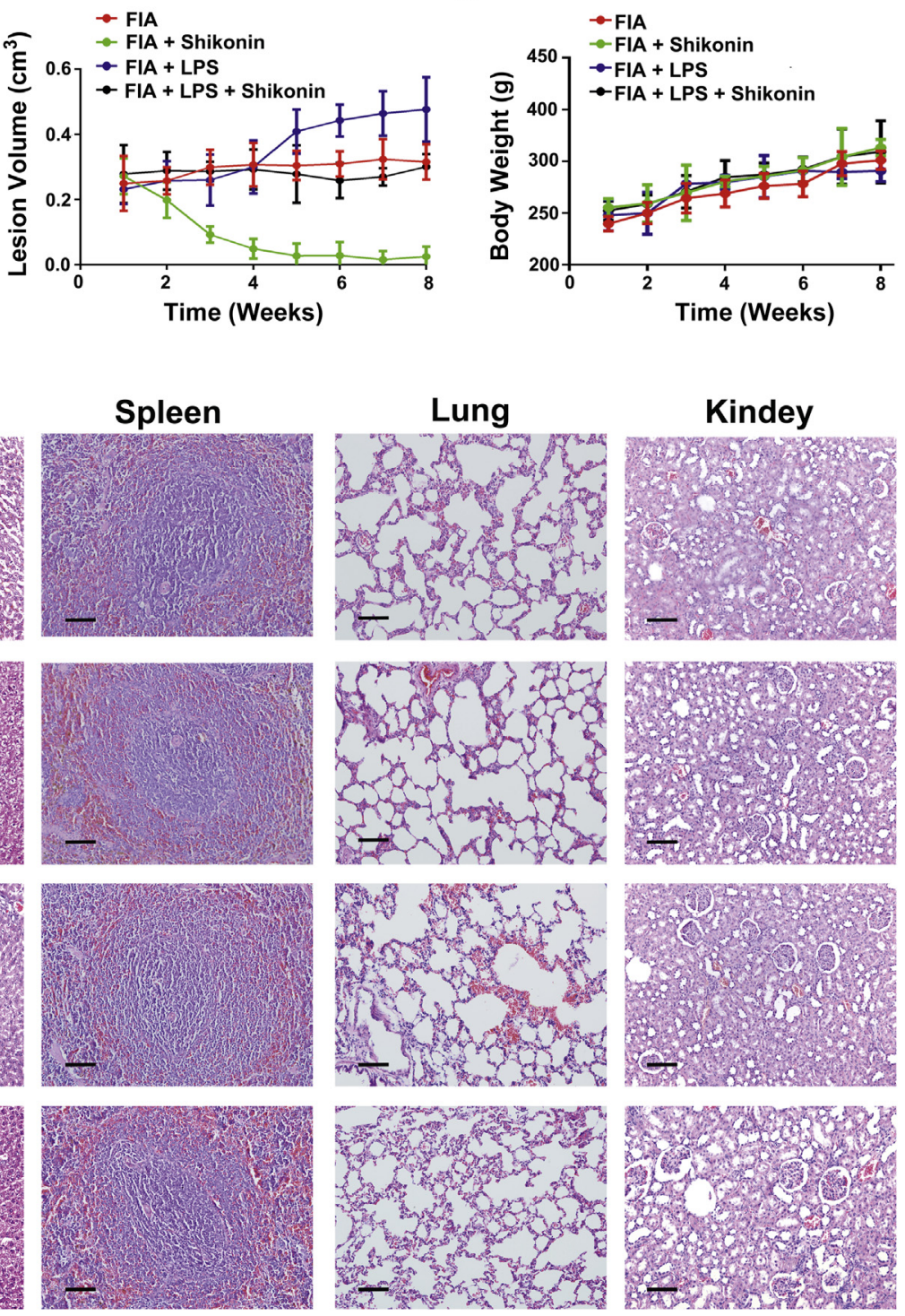

Figure 6 Inhibition of PKM2 suppresses the formation of LM lesions in a rat model. A: Representative images of LM lesions. Dotted lines indicate the boundaries of LM lesions in the rat neck. B: Lesion volumes and weights support the therapeutic effect of shikonin in the lesions both with and without infection. $\mathbf{C}$ and $\mathbf{D}$ : The body weights of rats (C) and important organic tissues staining by hematoxylin and eosin (D) after shikonin treatment indicated the safety of the drug administration. Data are expressed as means \pm SD. ${ }^{*} P<0.05,{ }^{* *} P<0.01$, and ${ }^{* * *} P<0.001 ;{ }^{\dagger} P<0.05,{ }^{\dagger \dagger} P<0.01$, and ${ }^{\dagger \dagger \dagger} P<0.001$ versus FIA. Scale bars: $1 \mathrm{~cm}$ (A); $50 \mu \mathrm{m}$ (D). FIA, Freund's incomplete adjuvant; LPS, lipopolysaccharide; PBS, phosphate-buffered saline.

have resulted in significant progress in treatment, they are still not ideal because they result in high recurrence rates. Moreover, basic investigation into the pathogenesis of LMs is rarely performed, especially the mechanism of rapid enlargement after infection or trauma.
Recent research ${ }^{26,27}$ has found somatic mutations in the PIK3CA gene present in LM-LECs, including E542K, E545K, and H1047R, which induce the activation of PIK3CA gene and regulate the proliferation and energy metabolism of LM-LECs. Glaser et $\mathrm{al}^{27}$ proposed that preferential selection by LM-LECs 
A

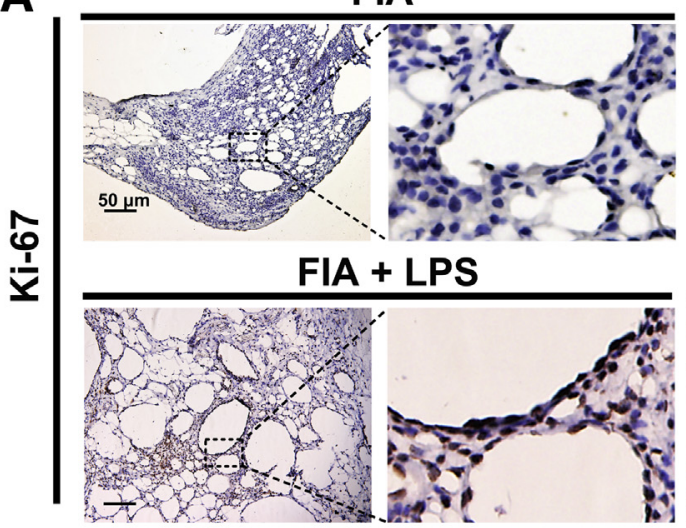



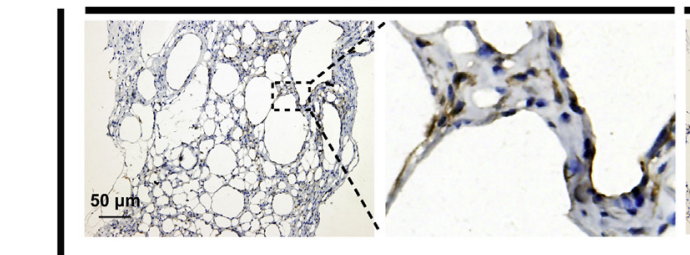

FIA
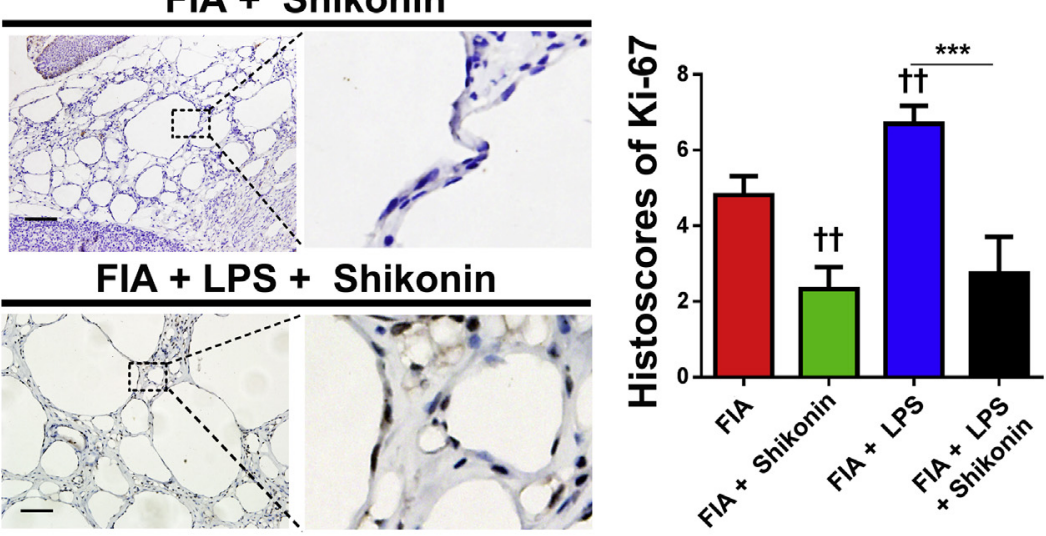

FlA + Shikonin
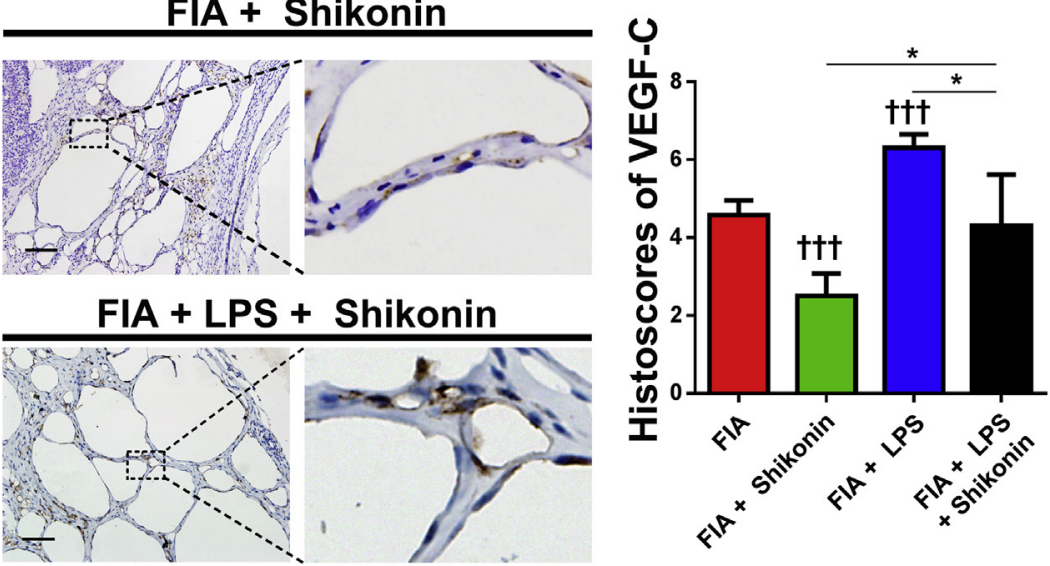

FIA + Shikinon

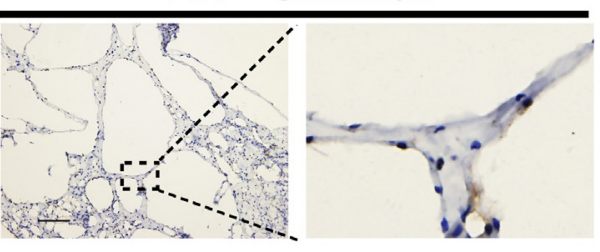

$\underset{\sum}{\stackrel{N}{\alpha}}$

FIA + LPS

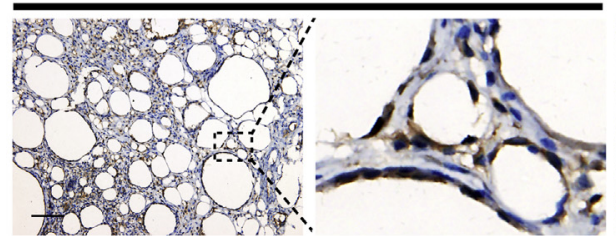

FIA + LPS + Shikinon
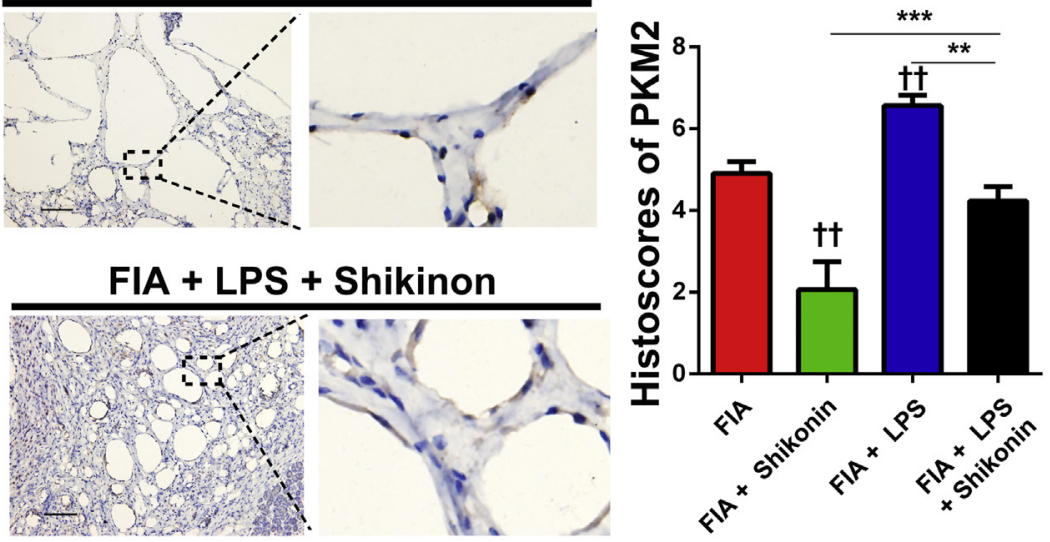

Figure 7 Immunohistochemical staining of Ki-67 (A), VEGF-C (B), and PKM2 (C) in LM lesions of rat models. The expressions of Ki67, VEGF-C and PKM2 were significantly up-regulated in infected LM lesions, which were induced by LPS, compared with noninfected LM lesions. After the treatment of Shikinon, the expression of these three proteins were obviously down-regulated either in noninfected lesions or in infected lesions. Data are expressed as means \pm SD. ${ }^{*} P<0.05,{ }^{* *} P<0.01$, and ${ }^{* *} P<0.001 ;{ }^{\dagger} P<0.01$, and ${ }^{{ }^{\dagger} \dagger} P<0.001$ versus FIA. Scale bars $=50 \mu \mathrm{m}$. FIA, Freund's incomplete adjuvant; LPS, lipopolysaccharide; PBS, phosphate-buffered saline.

of activated PIK3CA mutations may be relevant to the requirement of underlying metabolic defects in LM-LECs. These impairments affecting lipid management in LM-LECs, including trafficking, storage, and/or utilization of lipid, may be differentially accommodated by activated PIK3CA mutations, which directly switch LM-LECs from lipid utilization to lipid storage and promote glycolysis. In this study, PKM2 had a significantly higher expression in LM-LECs compared with lymphatic vessels in healthy skin tissue. Furthermore, the inhibition of PKM2 in the rat model of LM 
promoted lesion regression in vivo. These results demonstrate the importance of PKM2-mediated glycolysis in the pathogenesis of LMs.

Clinical investigation indicates that LMs usually grow slowly, but infections can significantly promote the progression of LMs. ${ }^{9}$ In a previous study in a rat model, LMs with local infection showed enhanced lymphangiogenesis, and the progression of LMs was accelerated with LPS. ${ }^{28}$ Consistent with findings from previous studies, ${ }^{9,10,28}$ the findings from present study indicate that the expression level of PKM2 was significantly up-regulated in LMs with infection compared with that in lesions without infection. As to the mechanism of PKM2 in promoting the progression of LMs, apart from its catalytic effect in glycolysis, PKM2 may also serve as protein kinase entering the nucleus to regulate behaviors of HDLEC. Previous studies have shown that PKM2 can translocate to the nucleus, where it directly interacts with hypoxia-inducible factor (HIF)-1 $\alpha$ to regulate the levels of proglycolytic enzymes. ${ }^{29,30}$ Furthermore, in a study by Corcoran and O'Neill, ${ }^{31}$ the level of PKM2 was enhanced with LPS stimulation to stabilize HIF1 $\alpha$, thereby regulating HIF1 $\alpha$ target genes, and accelerated the process of inflammatory diseases. Therefore, that PKM2 directly or indirectly mediates glycolysis might be a novel explanation of LM deterioration in inflammatory conditions.

Research on glycolysis has provided more options of targets for therapy. In the present study, PKM2 was selected as a target in the rat model of LM for several reasons. First, the PKM2-mediated Warburg effect has been widely detected in most cancer cells, especially in proliferating cells, which is unique from other metabolic catalytic enzymes. ${ }^{2,7}$ In the present study, PKM2 was elevated in LM tissues but was absent or weakly expressed in healthy skin tissues. Second, in contrast to the widely applied glucose analog 2-deoxy-D-glucose, which may cause severe adverse effects and systemic toxicity with its widely inhibiting glycolytic activity, ${ }^{32}$ shikonin, a specific small-molecular inhibitor of PKM2, previously used for the treatment of cirrhosis, psoriasis, and verruca plana, was safely and effectively used in both systemic and local administration. $^{14,33,34}$ In the present established rat model of LM, shikonin-treated lesions with or without inflection shrank gradually, and rats grew healthy. Therefore, targeting PKM2 offers a promising approach to LM treatment.

\section{Conclusion}

Taken together, this study first reports the potential role of PKM2-mediated glycolysis in the progress of LMs. Overexpression of PKM2 in LECs provides adequate substances and energy for proliferation, migration, and tubular formation. By establishing a rat model of LM, therapeutic efficacy and safety of shikonin in LM were obtained, reflecting the promising strategies of LM therapy. However, additional studies in LM-LECs are still needed for uncovering the precise mechanism.

\section{Author Contributions}

H.J. and Y.Z. performed the experiments and wrote the manuscript; H.J., X.L., and W.Z. analyzed and interpreted the data; S.Y. provided pathology support and help with analysis of the pathology data; X.Z. and D.M. provided technical support; J.Z. contributed intellectual content; W.Z. and Y.C. designed the study and wrote and revised the manuscript; all of the authors read and approved the final manuscript.

\section{Supplemental Data}

Supplemental material for this article can be found at http://doi.org/10.1016/j.ajpath.2020.10.003.

\section{References}

1. Yang W, Xia Y, Hawke D, Li X, Liang J, Xing D, Aldape K, Hunter T, Alfred Yung WK, Lu Z: PKM2 phosphorylates histone H3 and promotes gene transcription and tumorigenesis. Cell 2012, 150: 685-696

2. Lunt SY, Vander Heiden MG: Aerobic glycolysis: meeting the metabolic requirements of cell proliferation. Annu Rev Cell Dev Biol 2011, 27:441-464

3. Yu P, Wu G, Lee HW, Simons M: Endothelial metabolic control of lymphangiogenesis. Bioessays 2018, 40:e1700245

4. De Bock K, Georgiadou M, Schoors S, Kuchnio A, Wong BW, Cantelmo AR, et al: Role of PFKFB3-driven glycolysis in vessel sprouting. Cell 2013, 154:651-663

5. Teuwen LA, Geldhof V, Carmeliet P: How glucose, glutamine and fatty acid metabolism shape blood and lymph vessel development. Dev Biol 2019, 447:90-102

6. Kim B, Jang C, Dharaneeswaran H, Li J, Bhide M, Yang S, Li K Arany Z: Endothelial pyruvate kinase M2 maintains vascular integrity. J Clin Invest 2018, 128:4543-4556

7. Mazurek S: Pyruvate kinase type M2: a key regulator of the metabolic budget system in tumor cells. Int J Biochem Cell Biol 2011, 43: 969-980

8. Boscolo E, Coma S, Luks VL, Greene AK, Klagsbrun M, Warman ML, Bischoff J: AKT hyper-phosphorylation associated with PI3K mutations in lymphatic endothelial cells from a patient with lymphatic malformation. Angiogenesis 2015, 18:151-162

9. Yang JG, Sun YF, He KF, Ren JG, Liu ZJ, Liu B, Zhang W, Zhao YF: Lymphotoxins promote the progression of human lymphatic malformation by enhancing lymphatic endothelial cell proliferation. Am J Pathol 2017, 187:2602-2615

10. Zhong W, Jiang H, Zou Y, Ren J, Li Z, He K, Zhao J, Zhou X, Mou D, Cai Y: The YAP signaling pathway promotes the progression of lymphatic malformations through the activation of lymphatic endothelial cells. Pediatr Res 2020. [Epub ahead of print] doi:10. 1038/s41390-020-0863-0

11. Jia J, Cai Y, Wang R, Fu K, Zhao YF: Overexpression of allograft inflammatory factor- 1 promotes the proliferation and migration of human endothelial cells (HUV-EC-C) probably by up-regulation of basic fibroblast growth factor. Pediatr Res 2010, 67:29-34

12. Zhong WQ, Li ZZ, Jiang H, Zou YP, Wang HT, Cai Y, Zhao Y, Zhao JH: Elevated ATF4 expression in odontogenic keratocysts epithelia: potential involvement in tissue hypoxia and stromal M2 macrophage infiltration. J Histochem Cytochem 2019, 67:801-812

13. Sun Y, Jia J, Zhang W, Liu B, Zhang Z, Zhao Y: A reproducible invivo model of lymphatic malformation in rats. J Comp Pathol 2011, 145:390-398 
14. Andujar I, Recio MC, Giner RM, Rios JL: Traditional Chinese medicine remedy to jury: the pharmacological basis for the use of shikonin as an anticancer therapy. Curr Med Chem 2013, 20:2892-2898

15. Chen J, Xie J, Jiang Z, Wang B, Wang Y, Hu X: Shikonin and its analogs inhibit cancer cell glycolysis by targeting tumor pyruvate kinase-M2. Oncogene 2011, 30:4297-4306

16. Zhao X, Zhu Y, Hu J, Jiang L, Li L, Jia S, Zen K: Shikonin inhibits tumor growth in mice by suppressing pyruvate kinase M2-mediated aerobic glycolysis. Sci Rep 2018, 8:14517

17. Hong YK, Detmar M: Prox1, master regulator of the lymphatic vasculature phenotype. Cell Tissue Res 2003, 314:85-92

18. Schacht V, Ramirez MI, Hong YK, Hirakawa S, Feng D, Harvey N, Williams M, Dvorak AM, Dvorak HF, Oliver G, Detmar M: T1alpha/podoplanin deficiency disrupts normal lymphatic vasculature formation and causes lymphedema. EMBO J 2003, 22:3546-3556

19. Navarro A, Perez RE, Rezaiekhaligh MH, Mabry SM, Ekekezie II: Polarized migration of lymphatic endothelial cells is critically dependent on podoplanin regulation of Cdc42. Am J Physiol Lung Cell Mol Physiol 2011, 300:L32-L42

20. Verdegem D, Moens S, Stapor P, Carmeliet P: Endothelial cell metabolism: parallels and divergences with cancer cell metabolism. Cancer Metab 2014, 2:19

21. Beckert S, Farrahi F, Aslam RS, Scheuenstuhl H, Konigsrainer A, Hussain MZ, Hunt TK: Lactate stimulates endothelial cell migration. Wound Repair Regen 2006, 14:321-324

22. Ruan GX, Kazlauskas A: Lactate engages receptor tyrosine kinases Axl, Tie2, and vascular endothelial growth factor receptor 2 to activate phosphoinositide 3-kinase/Akt and promote angiogenesis. J Biol Chem 2013, 288:21161-21172

23. Yu P, Wilhelm K, Dubrac A, Tung JK, Alves TC, Fang JS, Xie Y, Zhu J, Chen Z, De Smet F, Zhang J, Jin SW, Sun L, Sun H, Kibbey RG, Hirschi KK, Hay N, Carmeliet P, Chittenden TW, Eichmann A, Potente M, Simons M: FGF-dependent metabolic control of vascular development. Nature 2017, 545:224-228

24. De Bock K, Georgiadou M, Carmeliet P: Role of endothelial cell metabolism in vessel sprouting. Cell Metab 2013, 18:634-647

25. Harris I, McCracken S, Mak TW: PKM2: a gatekeeper between growth and survival. Cell Res 2012, 22:447-449
26. Luks VL, Kamitaki N, Vivero MP, Uller W, Rab R, Bovee JV, Rialon KL, Guevara CJ, Alomari AI, Greene AK, Fishman SJ, Kozakewich HP, Maclellan RA, Mulliken JB, Rahbar R, Spencer SA, Trenor CC 3rd, Upton J, Zurakowski D, Perkins JA, Kirsh A, Bennett JT, Dobyns WB, Kurek KC, Warman ML, McCarroll SA, Murillo R: Lymphatic and other vascular malformative/overgrowth disorders are caused by somatic mutations in PIK3CA. J Pediatr 2015, 166:1048-1054.e1-5

27. Glaser K, Dickie P, Neilson D, Osborn A, Dickie BH: Linkage of metabolic defects to activated PIK3CA alleles in endothelial cells derived from lymphatic malformation. Lymphat Res Biol 2018, 16 : $43-55$

28. Zhang W, He KF, Yang JG, Ren JG, Sun YF, Zhao JH, Zhao YF: Infiltration of M2-polarized macrophages in infected lymphatic malformations: possible role in disease progression. Br J Dermatol 2016, $175: 102-112$

29. Luo W, Hu H, Chang R, Zhong J, Knabel M, O’Meally R, Cole RN, Pandey A, Semenza GL: Pyruvate kinase M2 is a PHD3-stimulated coactivator for hypoxia-inducible factor 1. Cell 2011, 145:732-744

30. Prangsaengtong O, Park JY, Inujima A, Igarashi Y, Shibahara N, Koizumi K: Enhancement of lymphangiogenesis in vitro via the regulations of HIF-1alpha expression and nuclear translocation by deoxyshikonin. Evid Based Complement Alternat Med 2013, 2013: 148297

31. Corcoran SE, O'Neill LA: HIF1alpha and metabolic reprogramming in inflammation. J Clin Invest 2016, 126:3699-3707

32. Voss M, Lorenz NI, Luger AL, Steinbach JP, Rieger J, Ronellenfitsch MW: Rescue of 2-deoxyglucose side effects by ketogenic diet. Int J Mol Sci 2018, 19:2462

33. Moon J, Koh SS, Malilas W, Cho IR, Kaewpiboon C, Kaowinn S, Lee K, Jhun BH, Choi YW, Chung YH: Acetylshikonin induces apoptosis of hepatitis B virus $\mathrm{X}$ protein-expressing human hepatocellular carcinoma cells via endoplasmic reticulum stress. Eur J Pharmacol 2014, 735:132-140

34. Yu YJ, Xu YY, Lan XO, Liu XY, Zhang XL, Gao XH, Geng L: Shikonin induces apoptosis and suppresses growth in keratinocytes via CEBP-delta upregulation. Int Immunopharmacol 2019, 72: $511-521$ 